\title{
Non-polio enteroviruses in faeces of children diagnosed with acute flaccid paralysis in Nigeria
}

\author{
T. O. C. Faleye ${ }^{1,2}$, M. O. Adewumi ${ }^{2}$, M. O. Japhet ${ }^{1,3^{*}}$, O. M. David', A. O. Oluyege ${ }^{1}$, J. A. Adeniji ${ }^{2,4}$ \\ and O. Famurewa ${ }^{1,5}$
}

\begin{abstract}
Background: The need to investigate the contribution of non-polio enteroviruses to acute flaccid paralysis (AFP) cannot be over emphasized as we move towards a poliovirus free world. Hence, we aim to identify non-polio enteroviruses recovered from the faeces of children diagnosed with AFP in Nigeria.

Methods: Ninety-six isolates, (95 unidentified and one previously confirmed Sabin poliovirus 3) recovered on RD cell culture from the stool of children $<15$ years old diagnosed with AFP in 2014 were analyzed. All isolates were subjected to RNA extraction, cDNA synthesis and three different PCR reactions (one panenterovirus 5'-UTR and two different VP1 amplification assays). VP1 amplicons were then sequenced and isolates identified.

Results: $92.71 \%$ (89/96) of the isolates were detected by at least one of the three assays as an enterovirus. Precisely, $79.17 \%$ (76/96), 6.25\% (6/96), 7.30\% (7/96) and 7.30\% (7/96) of the isolates were positive for both, positive and negative, negative and positive, as well as negative for both the 5'-UTR and VP1 assays, respectively. In this study, sixty-nine (69) of the 83 VP1 amplicons sequenced were identified as 27 different enterovirus types. The most commonly detected were CV-B3 (10 isolates) and EV-B75 (5 isolates). Specifically, one, twenty-four and two of the enterovirus types identified in this study belong to EV-A, EV-B and EV-C respectively.

Conclusions: This study reports the circulating strains of 27 non-polio enterovirus types in Nigerian children with AFP in 2014 and Nigerian strains of CV-B2, CV-B4, E17, EV-B80, EV-B73, EV-B97, EV-B93, EV-C99 and EV-A120 were reported for the first time. Furthermore, it shows that being positive for the $5^{\prime}-$ UTR assay should not be the basis for subjecting isolates to the VP1 assays.
\end{abstract}

Keywords: AFP, Enteroviruses, Nigeria, Non-polio enteroviruses, VP1 analysis

\section{Highlights}

- Identification of circulating enterovirus types in Nigerian children with AFP.

- Sequence data documented for 27 different enterovirus types circulating in Nigeria.

- First report of CV-B2, CV-B4, E17, EV-B80, EVB73, EV-B97, EV-B93, EV-C99 and EV-A120 in Nigeria.

\footnotetext{
* Correspondence: megdeoti@yahoo.com

${ }^{1}$ Department of Microbiology, Faculty of Science, Ekiti State University,

Ado-Ekiti, Ekiti State, Nigeria

${ }^{3}$ Department of Microbiology, Faculty of Science, Obafemi Awolowo

University, Ile-Ife, Osun State, Nigeria

Full list of author information is available at the end of the article
}

- Positivity for $5^{\prime}$-UTR assay should not be the basis for subjecting enterovirus isolates to VP1 assays.

- Results suggest importation of enterovirus clades, especially from Asia into Nigeria.

\section{Background}

Enteroviruses (EVs) are members of the genus Enterovirus in the family Picornaviridae, order Picornavirales. There are 13 species within the genus, four (EV-A to EV-D) of which (alongside the Rhinoviruses) have been repeatedly found to infect humans. The enterovirus capsid is a non-enveloped icosahedron with a diameter of 20-30 nM. It encloses a positive sense, single stranded RNA genome of $\sim 7500 \mathrm{nt}$. The genome has untranslated 
regions (5' and 3' UTRs) flanking a single one open reading frame (ORF), whose polyprotein product is auto-catalytically cleaved into eleven proteins; four structural (VP1 - VP4) and seven non-structural (2A $3 \mathrm{D})$ proteins. Amplification of the $5^{\prime}$-UTR and/or the VP1 region can be used to detect the presence of enteroviruses [1-5], and the nucleotide sequence of the VP1 gene is used to identify enterovirus isolates [1-6].

Poliovirus, the best studied member of the genus Enterovirus and the etiologic agent of poliomyelitis belongs to species $\mathrm{C}$ within the genus. Humans remain the only known host of poliovirus, thus suggesting feasibility of its eradication. Consequently, in 1988, the World Health Assembly (WHA) resolved to eradicate poliomyelitis by the year 2000 [7], and the Global Polio Eradication Initiative (GPEI) was established. Courtesy of the GPEI's activities, by year 2015 indigenous poliovirus transmission has been interrupted globally except in Pakistan and Afghanistan (www.polioeradication.org). This success has been the result of effective vaccination and intensive surveillance. Two poliovirus vaccine preparation (Oral Polio Vaccine [OPV] and Inactivated Polio Vaccine [IPV]) are currently being used by the GPEI and both are very effective [8]. However, due to the reversion of OPV, as part of the 'end game' strategy, the GPEI is tilting towards IPV as we approach the final phase of polio eradication [9].

Coupled with the vaccination effort is a very effective surveillance network that looks for polioviruses in both sewage-contaminated water (Environmental Surveillance [ES]) and children below the age of 15 years diagnosed with AFP. The ES strategy searches for enteroviruses in sewage-contaminated water due to the fact that all enterovirus infected individuals shed the virus in large amounts in faeces for several weeks and in turn into sewage and/or sewage contaminated water [10] . Therefore, ES is very sensitive and can detect enterovirus isolates from both symptomatic and asymptomatic individuals. The demerit of ES based strategy is that, alone, it cannot differentiate which isolates are associated with clinical manifestations and hospitalisations. On the other hand, AFP surveillance finds enteroviruses associated with a clinical manifestations and consequent hospitalisation. However, considering that AFP surveillance detects only the $<10 \%$ of enterovirus infections with clinical manifestations, the inability of AFP surveillance to see beyond the tip of the iceberg is the strength of the ES surveillances strategy. Therefore, combining both strategies better illuminates our understanding of the epidemiological and evolutionary trajectory of enteroviruses, particularly with respect to pathogenicity. Hence, the reason the ES-AFP surveillance strategies are combined by the Global Polio Eradication initiative (GPEI) in some countries [11, 12].
As part of the surveillance network are about 150 laboratories globally (Global Polio Laboratory Network [GPLN]) that use the RD-L20B isolation protocol [11, 12] for poliovirus isolation. The RD-L20B isolation protocol is predicated on two different cell lines, RD (from a striated muscle cancer) [13] and L20B (a murine transgenic L cell that expresses the poliovirus receptor and is consequently permissive and susceptible to polioviruses) [14, 15]. As a by-product of the poliovirus surveillance programme, the GPLN recovers several non-polio enteroviruses (NPEVs) on the RD cell line.

The earliest molecular epidemiology study documenting NPEV from AFP cases in Nigeria was carried out using the RD-L20B protocol between 2002 and 2004 [16, 17]. Subsequent studies [18-22] generating nucleotide sequence data on enterovirus diversity in Nigeria have been largely ES based. The only exception is a recent study [23] on enterovirus diversity in healthy Nigerian children, in which a cell-culture independent enterovirus detection strategy was employed. Consequently, in this study we revisit NPEVs from AFP cases in Nigeria in an attempt to revise their diversity in individuals with this clinical condition in the region.

\section{Methods \\ Samples}

Ninety-six (96) RD cell culture isolates were analysed in this study. These isolates were recovered from the faeces of children below the age of 15 years that were diagnosed with AFP. A total of 8786 samples were received by the polio lab from January to June 2014, 7005 of which had no viral detection. Isolates were recovered from 1781 samples, 1024 of which were non-poliovirus isolates. Hence, the non-poliovirus isolates we attempted to characterize in this study represent $9.3 \%(95 / 1024)$ of those recovered by the polio lab during the period. The stool samples from which these isolates were recovered were collected in accordance with the national ethical guidelines as part of the National AFP surveillance programme in Nigeria and sent to the WHO National Polio Laboratory in Ibadan, Nigeria (subsequently referred to as the polio lab) to ascertain whether poliovirus is the etiologic agent of the diagnosed AFP using the WHO algorithm [12]. The isolates analyzed in this study were subsequently anonymized for further studies.

The WHO algorithm stipulates that fecal suspension from all AFP cases be inoculated into RD and L20B cell lines. Isolates that show cytopathic effect (CPE) on both cell lines are considered to be polioviruses, and subsequently subjected to intratypic differentiation (ITD) to differentiate between the wild type and the vaccine strain. On the other hand, isolates that only show CPE in RD cell line are assumed to be 
non-polio enteroviruses and stored away since they are not of 'urgent' programmatic importance to the GPEI.

To assemble the 96 isolates analyzed in this study, from the archives, sixteen (16) isolates that showed CPE in RD cell line only, were randomly selected each month from January to May 2014. In June, fifteen (15) isolates were selected, and a previously identified Sabin strain poliovirus 3 was added to serve as control for the study. Besides this known isolate, none of the other 95 isolates had been previously identified.

\section{RNA extraction and CDNA synthesis}

The algorithm followed in this study is as depicted in Fig. 1. Using the RNA extraction kit (Jena Bioscience, Jena, Germany), RNA was extracted from the isolates according to the manufacturer's instructions. Script cDNA Synthesis Kit (Jena Bioscience, Jena, Germany) was used for complementary DNA (cDNA) synthesis as instructed by the manufacturer. Specifically, random hexamers were used for cDNA synthesis as previously described [20]. The cDNA was then stored at $-80{ }^{\circ} \mathrm{C}$ and used for all polymerase chain reaction (PCR) assays.

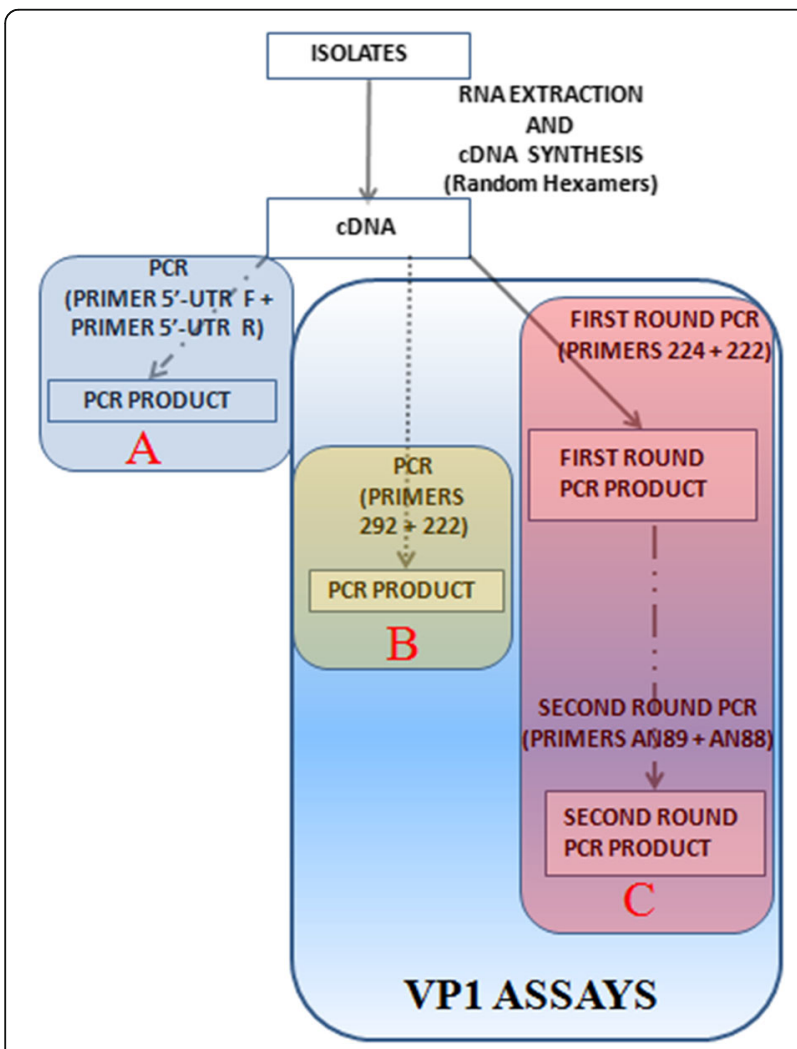

Fig. 1 The algorithm used in this study. A depicts the 5'-UTR assay. $B$ and $C$ show the two VP1 assays. While B has only one stage of $\mathrm{PCR}, \mathrm{C}$ has two consecutive stages (snPCR) of PCR

\section{Polymerase chain reaction (PCR) assays}

As shown in the algorithm for this study (Fig. 1), three different PCR assays were run; one $5^{\prime}$-UTR and two VP1 assays. The $5^{\prime}$-UTR and first PanEnterovirus VP1 PCR (PE-VP1-PCR) were one-step PCR assays. The second PanEnterovirus VP1 PCR was a semi-nested PCR assay (PE-VP1-snPCR) and was used to amplify the partial VP1 gene in those isolates for which the PE-VP1PCR was negative. The 5' -UTR assay targets the 5'untranslated region which is very conserved but cannot be used to determine enterovirus type. The remaining two assays both target the highly variable VP1 region which is protein coding and encodes one of the capsid proteins. Unlike the 5'UTR, nucleotide sequence of the VP1 region can be and is used for enterovirus type determination [1-6]. It is essential to note, as mentioned above, that one of the VP1 assays is a one-step RT-PCR assay while the other is an RT- semi-nested PCR assay. Only the VP1 amplicons generated were sequenced and subsequently used for enterovirus type determination (Fig. 1).

All primers were made in $100 \mu \mathrm{M}$ concentrations and all PCR assays were carried out in $30 \mu \mathrm{L}$ reaction volumes. All PCR products were resolved on $2 \%$ agarose gels stained with ethidium bromide and viewed using a UV transilluminator.

\section{5'-UTR PCR and PanEnterovirus VP1 PCR (PE-VP1-PCR)}

Primers panent $5^{\prime}$-UTR F and panent $5^{\prime}$-UTR R [12] were used for the $5^{\prime}$-UTR PCR assay while primers 229 and $222[2,6]$ were used for the PE-VP1-PCR assay. Each $30 \mu \mathrm{L}$ reaction contained $6 \mu \mathrm{L}$ of Red Load Taq (Jena Bioscience), $5 \mu \mathrm{L}$ of cDNA, $0.3 \mu \mathrm{L}$ of each primer and $18.4 \mu \mathrm{L}$ of RNase-free water. Thermal cycling was done as follows: $94{ }^{\circ} \mathrm{C}$ for $3 \mathrm{~min}, 45$ cycles of denaturation at $94{ }^{\circ} \mathrm{C}$ for $30 \mathrm{~s}$, annealing at $42{ }^{\circ} \mathrm{C}$ for $30 \mathrm{~s}$, and extension at $60{ }^{\circ} \mathrm{C}$ for $30 \mathrm{~s}$ with ramp of $40 \%$ from $42{ }^{\circ} \mathrm{C}$ to $60{ }^{\circ} \mathrm{C}$. This was followed by $72{ }^{\circ} \mathrm{C}$ for $7 \mathrm{~min}$, and thereafter the sample was held at $4{ }^{\circ} \mathrm{C}$ until the reaction was terminated.

\section{PanEnterovirus VP1 semi-nested PCR (PE-VP1-snPCR)}

This assay is a semi-nested PCR assay. Primers 224 and $222[5,6]$ were used for the first round PCR while primers AN89 and AN88 [5, 6] were used for the second round PCR assay. For the first round PCR assay, the $30 \mu \mathrm{L}$ reaction contained $6 \mu \mathrm{L}$ of Red Load Taq (Jena Bioscience), $5 \mu \mathrm{L}$ of cDNA, $0.3 \mu \mathrm{L}$ of each primer and $18.4 \mu \mathrm{L}$ of RNase-free water. Thermal cycling was done as follows: $94{ }^{\circ} \mathrm{C}$ for $3 \mathrm{~min}, 45$ cycles of denaturation at $94{ }^{\circ} \mathrm{C}$ for $30 \mathrm{~s}$, annealing at $42{ }^{\circ} \mathrm{C}$ for $30 \mathrm{~s}$, and extension at $60{ }^{\circ} \mathrm{C}$ for $60 \mathrm{~s}$ with ramp of $40 \%$ from $42{ }^{\circ} \mathrm{C}$ to $60{ }^{\circ} \mathrm{C}$. This was followed by $72{ }^{\circ} \mathrm{C}$ for $7 \mathrm{~min}$, and thereafter the sample was held at $4{ }^{\circ} \mathrm{C}$ until the reaction was terminated. The conditions were 
the same for the second round PCR assay except for following modifications: Instead of cDNA, the product of the first round PCR assay was used as template for the second round assay. Also, the extension time for the second round assay was $30 \mathrm{~s}$ as opposed to $60 \mathrm{~s}$ for the first round assay.

\section{Amplicon sequencing and enterovirus typing}

Only amplicon of positive PCR reactions for the two VP1 PCR assays (PE-VP1-PCR and PE-VP1-snPCR) were sequenced using the respective forward and reverse primers for each of the assays. Amplicons were shipped to Macrogen Inc., Seoul, South Korea, for purification and sequencing. Subsequently, enterovirus genotype and species were determined using the Enterovirus Genotyping Tool [24].

\section{Phylogenetic analysis}

The CLUSTAL W program in MEGA 5 software [25] was used with default settings to align sequences of four (the two most commonly isolated and the two for which most extensive nucleotide sequence data from the region exists) of the enterovirus serotype described in this study alongside those retrieved from GenBank. Subsequently, a neighbor-joining tree was constructed using the same MEGA 5 software with the Kimura-2 parameter model [26] and 1000 bootstrap replicates. The accession numbers of sequences retrieved from GenBank for this analysis are indicated in the sequence names on the phylograms.

\section{Nucleotide sequence accession numbers}

All sequences reported in this study have been deposited in GenBank and assigned accession numbers KX580638 KX580702 and KX656918 - KX656912.

\section{Ethics approval}

For clarity sake it is crucial to re-iterate that enterovirus isolates were analysed in this study. The stool samples from which these isolates were recovered were collected in accordance with the national ethical guidelines as part of the National AFP surveillance programme in Nigeria and sent to the WHO National Polio Laboratory in Ibadan, Nigeria to ascertain whether poliovirus is the etiologic agent of the diagnosed AFP using the WHO algorithm [12]. The isolates analyzed were subsequently anonymized for further studies before use in this study. Thus, this article does not contain any studies with human participants performed by any of the authors. In addition, no information that can be used to associate the isolates analyzed in this study to any stool samples or the individual from which they were collected is included in this manuscript.

\section{Results}

\section{5'-UTR assay}

Precisely, $85.42 \%$ (82/96) of the isolates analyzed, including the previously identified Sabin 3, had the expected $\sim 114 \mathrm{bp}$ band size and were consequently positive for the 5 '-UTR PCR screen (Table 1).

\section{VP1 Assays (PE-VP1-PC and PE-VP1-snPCR)}

Fifty-nine (59) of the samples analyzed in this study, excluding the previously identified Sabin 3, had the $\sim 350$ bp expected band size and were consequently positive for the PE-VP1-PCR screen. The remaining thirtyseven (37) samples were negative for the PE-VP1-PCR screen.

Of the remaining thirty-seven (37) samples, twentyfour (24), including the previously identified Sabin 3, had the $\sim 350$ bp expected band size and were thereby positive for the PE-VP1-snPCR screen. The remaining 13 samples were negative for the PE-VP1-snPCR screen.

Altogether, $86.46 \%$ (83/96) of the isolates, including the previously identified Sabin 3, had the expected $\sim 350$ bp band size and were consequently positive for the VP1 assays. The remaining $13.54 \%$ (13/96) were negative for the VP1 assays (Table 1).

\section{5'-UTR versus VP1 assays}

Overall, $92.71 \%(89 / 96)$ of the isolates were detected by at least one of the three assays as an enterovirus. Precisely, $79.17 \%(76 / 96)$ of the isolates were positive for both the $5{ }^{\prime}$-UTR and VP1 assays. Furthermore, 6.25\% $(6 / 96)$ of the isolates were positive for the 5 '-UTR assay alone, $7.30 \%$ (7/96) were positive for only VP1 assays and $7.30 \%(7 / 96)$ of the isolates were negative for both the $5^{\prime}$-UTR and VP1 assays (Table 1).

\section{Enterovirus typing}

Sixty-nine (69) of the 83 amplicons generated from the VP1 PCR assays and successfully sequenced were identified. The remaining 14 could not be typed due to the presence of multiple peaks in their electropherograms. Twenty-seven different enterovirus types were identified from the 69 exploitable amplicons (Table 2). Specifically, one (1), twenty-four (24) and two (2) of the enterovirus types identified in this study belong to EV-A, EV-B and EV-C respectively (Table 2 ).

\section{Phylogenetic analysis}

Of the different enterovirus types identified in this study, only four (Coxsackievirus B3 [CV-B3] and EV-B75 [the two most commonly isolated] and Echovirus 7 [E7] and E19 [the two for which most extensive nucleotide sequence data from the region exists]) were subjected to phylogenetic analysis. The CV-B3 tree (Fig. 2), showed 
Faleye et al. Virology Journal (2017) 14:175

Page 5 of 14

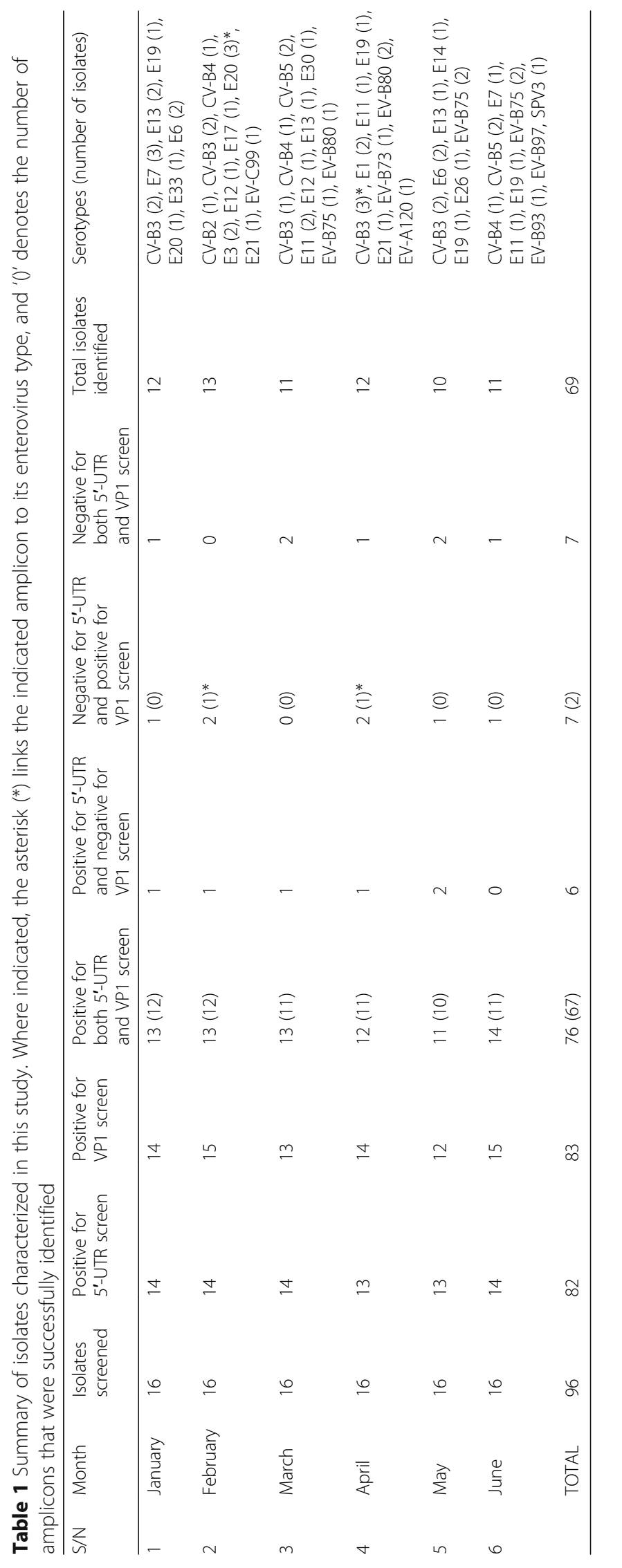


Table 2 Summary of enterovirus serotypes identified in this study

\begin{tabular}{|c|c|c|c|c|}
\hline$S / N$ & Types & Months detected & $\begin{array}{l}\text { Number of } \\
\text { isolates }\end{array}$ & $\begin{array}{l}\text { Cumulative } \\
\text { number }\end{array}$ \\
\hline & EV-A & & & \\
\hline \multirow[t]{2}{*}{1} & EV-A120 & Apr (1) & 1 & 1 \\
\hline & \multicolumn{4}{|l|}{ EV-B } \\
\hline 2 & CV-B2 & Feb (1), & 1 & 2 \\
\hline 3 & CV-B3 & $\begin{array}{l}\text { Jan (2), Feb (2), } \\
\text { Mar (1), Apr (3), } \\
\text { May (2) }\end{array}$ & 10 & 12 \\
\hline 4 & CV-B4 & $\begin{array}{l}\text { Feb (1), Mar (1), } \\
\text { Jun (1) }\end{array}$ & 3 & 15 \\
\hline 4 & CV-B5 & $\operatorname{Mar}(2), \operatorname{Jun}(2)$ & 4 & 19 \\
\hline 6 & E1 & Apr (2) & 2 & 21 \\
\hline 7 & E3 & Feb (2) & 2 & 23 \\
\hline 8 & E6 & Jan (2), May (2) & 4 & 27 \\
\hline 9 & E7 & Jan (3), Jun (1) & 4 & 31 \\
\hline 10 & E11 & $\begin{array}{l}\text { Mar (2), Apr (1), } \\
\text { Jun (1) }\end{array}$ & 4 & 35 \\
\hline 11 & E12 & Feb (1), Mar (1) & 2 & 37 \\
\hline 12 & E13 & $\begin{array}{l}\text { Jan (2), Mar (1), } \\
\text { May (1) }\end{array}$ & 4 & 41 \\
\hline 13 & E14 & May (1) & 1 & 42 \\
\hline 14 & E17 & Feb (1) & 1 & 43 \\
\hline 15 & E19 & $\begin{array}{l}\text { Jan (1), Apr (1), } \\
\text { May (1), June (1) }\end{array}$ & 4 & 47 \\
\hline 16 & E20 & Jan (1), Feb (3) & 4 & 51 \\
\hline 17 & E21 & Feb (1), Apr (1) & 2 & 53 \\
\hline 18 & E26 & May (1) & 1 & 54 \\
\hline 19 & E30 & $\operatorname{Mar}(1)$ & 1 & 55 \\
\hline 20 & E33 & $\operatorname{Jan}(1)$ & 1 & 56 \\
\hline 21 & EV-B73 & Apr (1) & 1 & 57 \\
\hline 22 & EV-B75 & $\begin{array}{l}\text { Mar (1), May (2), } \\
\text { Jun (2) }\end{array}$ & 5 & 62 \\
\hline 23 & EV-B80 & $\operatorname{Mar}(1), \operatorname{Apr}(2)$ & 3 & 65 \\
\hline 24 & EV-B93 & Jun (1) & 1 & 66 \\
\hline 25 & EV-B97 & Jun (1) & 1 & 67 \\
\hline & \multicolumn{4}{|l|}{ EV-C } \\
\hline 26 & EV-C99 & Feb (1) & 1 & 68 \\
\hline 27 & SPV3 & Jun (1) & 1 & 69 \\
\hline
\end{tabular}

that the lineage of the CV-B3 genotype detected in 2002 is different from the lineage detected in 2014. The CVB3 isolates described in this study belong to the same lineage as those circulating in Niger; a country also in West-Africa which shares border with Nigeria in the North. In addition, it is important to note that the CVB3 sequences detected in this study appear to share a distant common ancestor with those from Asia (Fig. 2).

For the EV-B75, two (sub lineages 1 and 3) different but closely related genotypes were detected. Both were however related to EV-B75 isolates detected in Finland in 2004 (sub lineage 2). Two of the EV-B75 isolates recovered in this study (sub lineage 1) share a common ancestor with a cluster found circulating in Niger, Gambia and Guinea. The other two EV-B75 isolates described in this study, (sub lineage 3) share a common ancestor with an isolate recovered from sewage contaminated water collected in Northern Nigeria in 2012 and an EV-B75 isolate recovered from a child with AFP in Niger (Fig. 3).

For the E19, two different genotypes were detected. Both were also very different from the E19 strains isolated in 2003 (SSA 1; sub lineage 1). The lineages to which the isolates belong are indicated as SSA 2; sub lineages 1 and 6 (Fig. 4). The single isolate from this study in SSA 2; sub lineage 1 shares a common ancestor with a lineage that was repeatedly isolated from sewage contaminated water in Lagos, Southwestern Nigeria since 2010. The remaining three strains however, belong to SSA 2; sub lineage 6 and are more closely related to an ES isolate recovered in Kano State, Northern Nigeria in 2012 and an isolate recovered from a child with AFP in Niger in 2014 (Fig. 4). The E19 isolates recovered from children with AFP in Gambia and Senegal belong to SSA 2; sub lineage 4 while that from Guinea belong to SSA 1; sub lineage 3.

As regards, E7, two different genotypes were also detected; SSA sub lineage A2 and the globally circulating lineage (Fig. 5). Two of the isolates from this study in SSA sub lineage A2 share a common ancestor with a lineage that was first isolated from sewage contaminated water in Lagos, Southwestern Nigeria in 2010. The remaining two strains however, belong to the global lineage and share a common ancestor with isolates repeatedly recovered from AFP cases in India since 2008 (Fig. 5).

\section{Discussion}

In this study, we document nucleic acid sequence data for twenty-seven (27) different enterovirus types circulating and particularly present in children below the age of 15 years diagnosed with AFP in Nigeria in 2014. It is important to mention that prior this study, no nucleic acid sequence data existed in Genbank for nine (9) of these enterovirus types from Nigeria. To be precise, to the best of the authors' knowledge, nucleic acid sequence data for Nigerian strains of E17, CV-B2, CV-B4, EV-B97, EVB80, EV-B73, EV-B93, EV-C99 and EV-A120 are being reported for the first time. It is however, our opinion, that the fact that these molecular sequence data are being reported for the first time does not imply new introduction of these types into the region. Rather, we believe that these types had probably been around for a long time. Hence not detecting them might reflect the lack of 


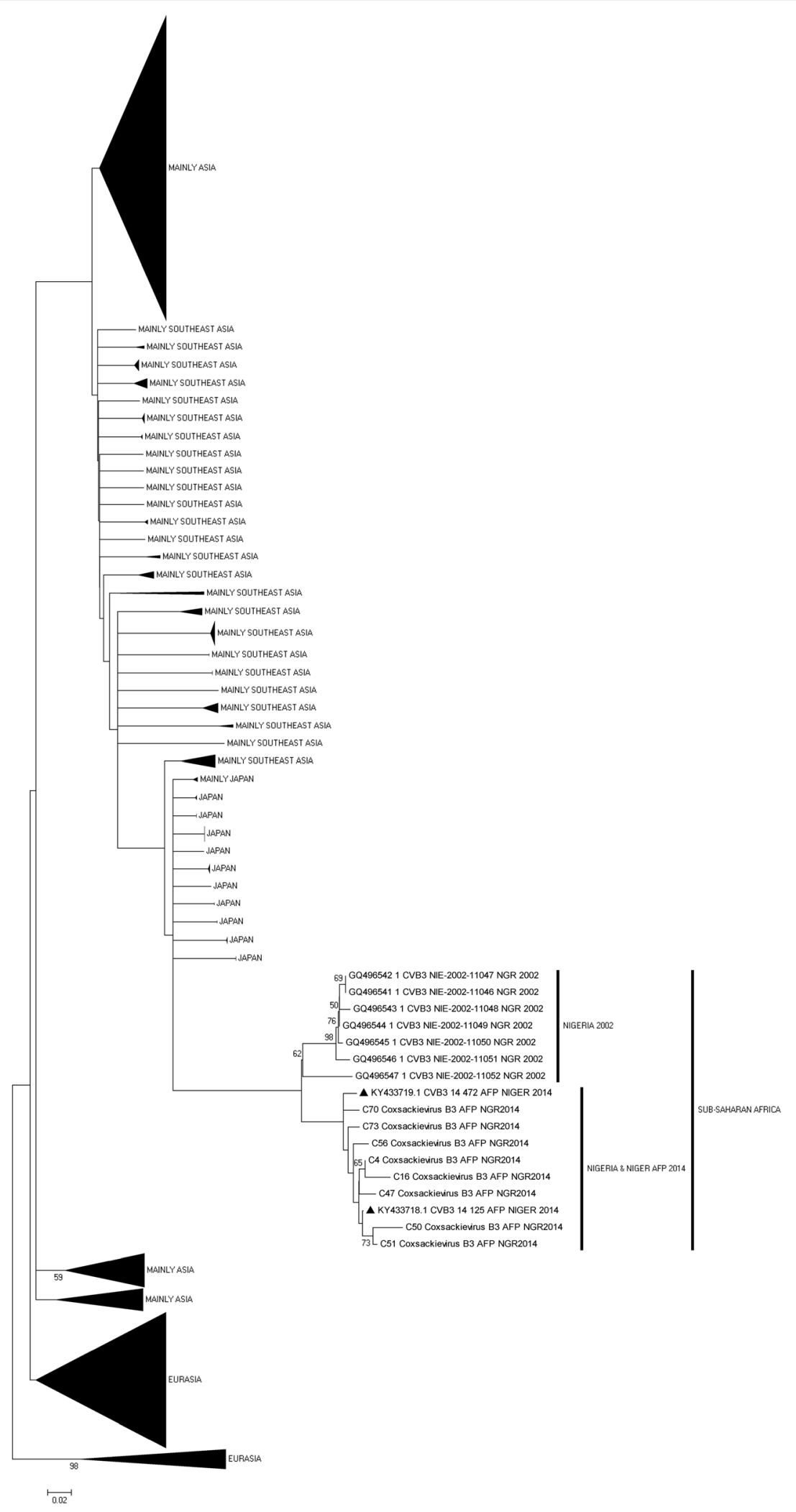

Fig. 2 (See legend on next page.) 
(See figure on previous page.)

Fig. 2 Phylogram of genetic relationship between VP1 nucleotide sequences of CV-B3 isolates. The phylogenetic tree is based on an alignment of the partial VP1 sequences. The CV-B3 sequences recovered in Nigeria in 2002 and the strains newly described in this study are indicated within sub-Saharan Africa (SSA). The strains indicated with black triangl represent CV-B3 strains from Niger; a country in west-Africa that shares a border with Northern-Nigeria

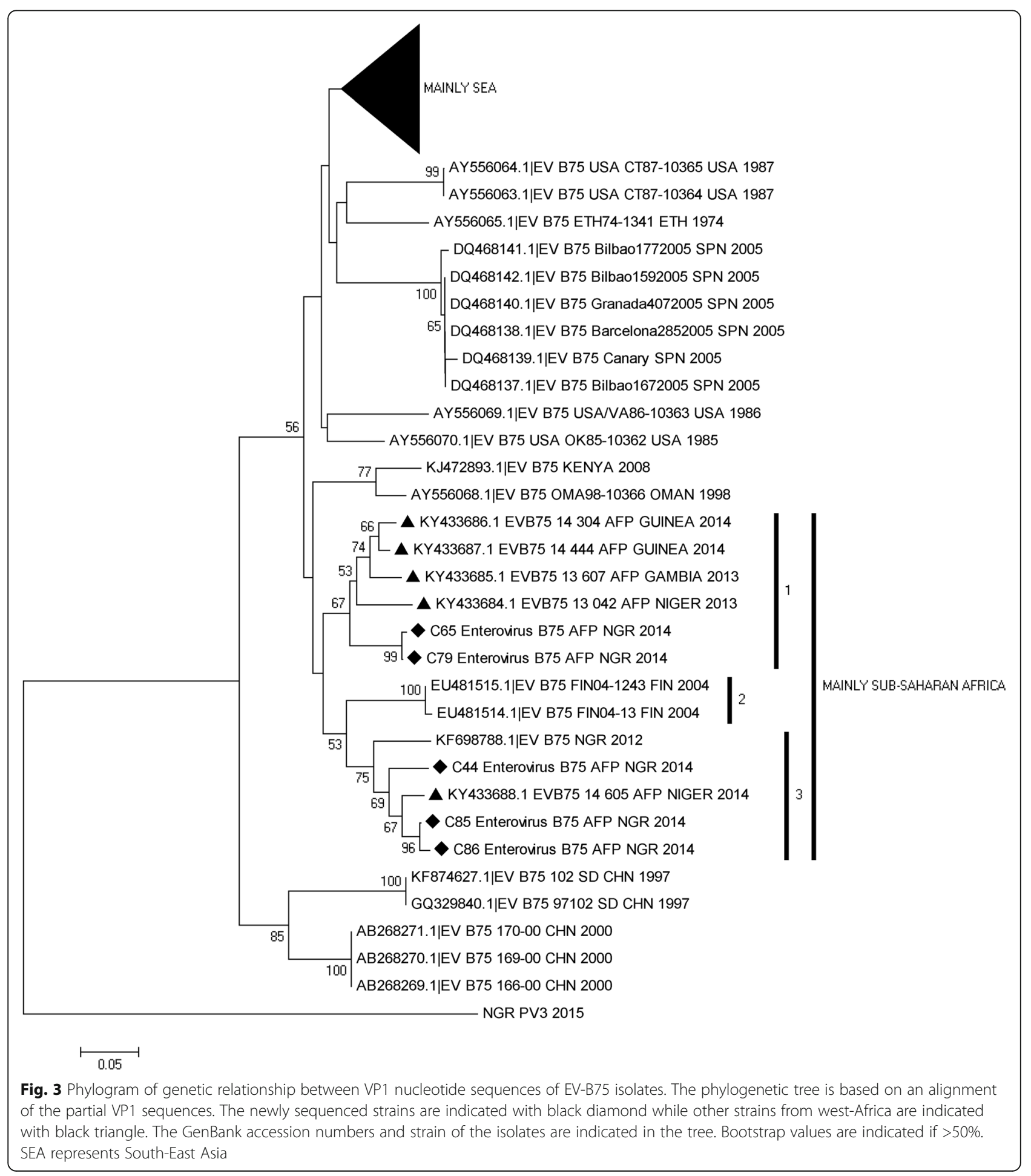




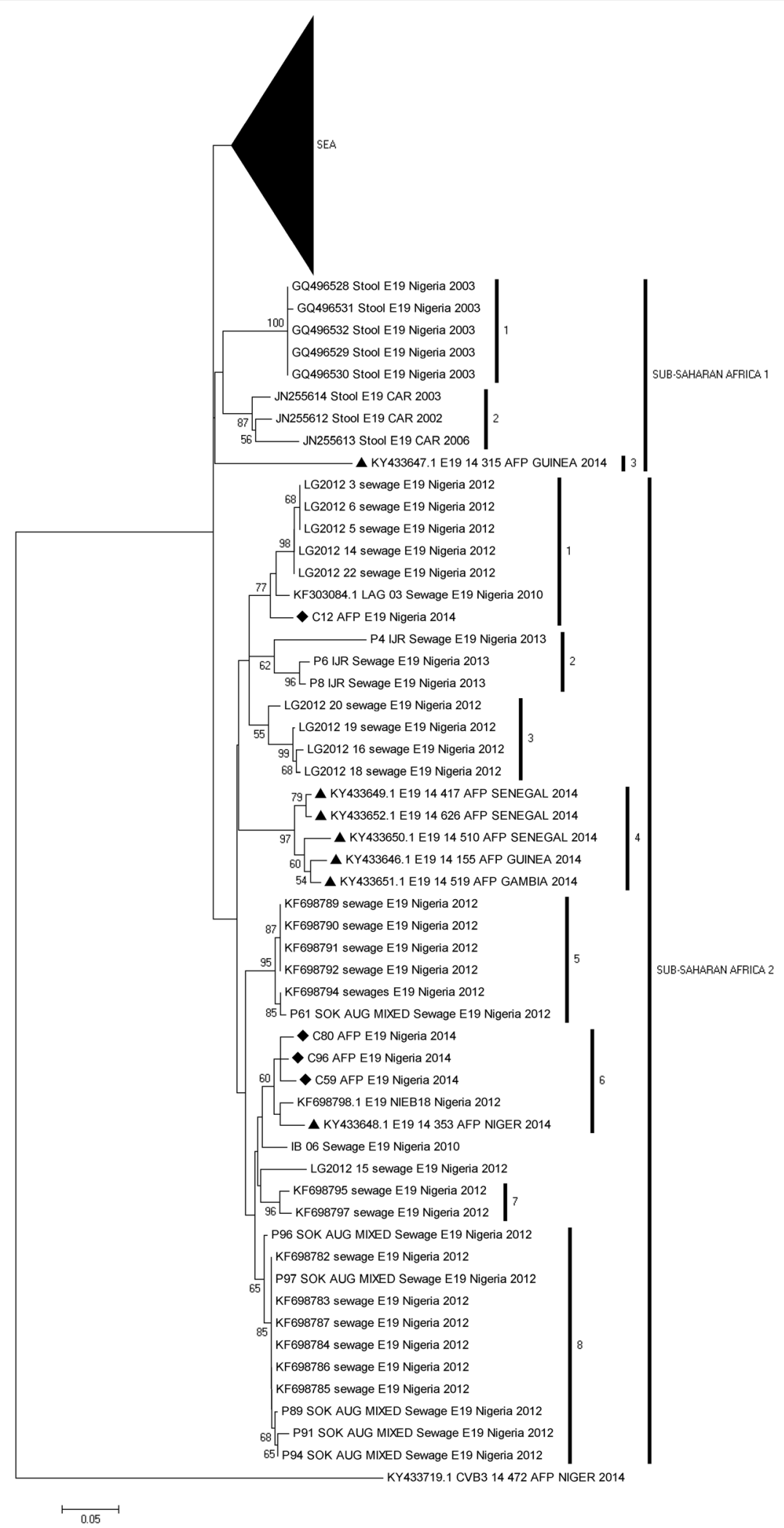

Fig. 4 (See legend on next page.) 
(See figure on previous page.)

Fig. 4 Phylogram of genetic relationship between VP1 nucleotide sequences of E19 isolates. The phylogenetic tree is based on an alignment of the partial VP1 sequences. The newly sequenced strains are indicated with black diamond while other strains from west-Africa are indicated with black triangle. The GenBank accession numbers and strain of the isolates are indicated in the tree. Bootstrap values are indicated if $>50 \%$. SEA represents South-East Asia

interest in NPEVs because the global effort focused on eradicating polioviruses. However, as the goal of poliovirus eradication nears [9], there might be an upsurge of interest in NPEVs in a bid to better understand enterovirus biology and association with varying clinical conditions.

In this study, of the 69 isolates sequenced, $95.65 \%$ (66/69) belong to HEV-B representing $88.9 \%$ (24/27) of enterovirus types identified. (Table 2). This is consistent with the findings of other studies from the region $[16,17,27]$ predicated on the RD-L20B algorithm, irrespective of whether healthy children [27] or those diagnosed with AFP were investigated [16, 17]. However, it was recently shown that when cell-culture bias is bypassed by the direct detection of enteroviruses from stool specimen, EV-As appear to be the most preponderant in the stool of healthy children from the region [23]. Furthermore, recent studies [20, 21] showed that most CV-A13s (EV-Cs) circulating in the region selectively replicate with evident cytopathology, and can be isolated on MCF-7, but not in RD cell line. Also, when the same sample was simultaneously inoculated into RD and MCF-7 cell lines, EV-Bs and EV-Cs respectively are specifically recovered on the two cell lines [21]. In fact, we recently observed [28] that when AFP samples that are negative for enteroviruses using the RD-L20B enterovirus detection algorithm [11] are subjected to the recently recommended [6] direct detection RT-snPCR algorithm described by Nix et al., [5], EV-Cs form the majority of enteroviruses detected. In addition, we also recently observed (unpublished) that about $50 \%$ of stool suspension from children with AFP in the region that yield EV-Bs in $\mathrm{RD}$ cell line also have an EV-C member present in the faecal suspension that was not detectable by the cell line. Put together, the preponderance of EV-B depicted by the results of this study and previous RD-L20B based studies from the region and globally should not be interpreted as an unbiased picture of the diversity landscape of enteroviruses in the AFP samples that yielded the isolates analyzed. Rather, it should be correctly viewed as the landscape as seen through the bias of RD cell culture.

Though in this study we describe enteroviruses present in the stool of children diagnosed with AFP, the findings of this study show the significance of merging ES and AFP data. Phylogenetic analysis (Figs. 3, 4 and 5) suggests that representatives of some of the lineages of EVB75, E19 and E7 detected in the AFP cases had been previously detected through environmental surveillance. Interestingly, all the sequences from Nigeria in Figs. 4 and 5 except those from this study and the 2002-2004 sequences were from environmental surveillance. The ES data rightly show that more lineages are circulating in the population than depicted by the AFP data reported in this study. This thereby emphasizes the power of ES to illuminate our understanding of the diversity of enterovirus types and lineages circulating in the population. This will, consequently, further enable us to better understand the evolutionary trajectory of these enterovirus types and detect their silent circulation especially in the absence of clinical manifestations.

Surveillance of enterovirus diversity among healthy children can also increase the power of the ES-AFP surveillance strategy. For instance, it was previously shown [23] that other enterovirus types not detected in this study (e.g. EV-A71 and several CV-A types) were also present and circulating in Nigeria in the same year, 2014. In addition, we had shown [23] that the EV-B80 detected in a healthy Nigerian child belonged to the same lineage as those detected in children diagnosed with AFP in this study. We further showed [23] that the EV-C99 lineage found in a healthy Nigerian child is different from that described in this study. Thus, though not suggested or included in the GPEI surveillance algorithm, enterovirus surveillance in healthy children is useful and can significantly complement the ES-AFP strategy of the GPEI.

The baseline nucleotide sequence data for CV-B3 and E19 circulating in the region were generated in 2002 and 2003 respectively $[16,17]$. Hence, the data presented in this study is a re-sampling of circulating strains of these types after over a 10-year period. For both types, the strains circulating when the baseline data was generated appear to have been completely replaced (Figs. 2 and 4). On the other hand, E7 and EV-B75 for which baseline sequence data from the region was generated in 2010 [18] and 2012 [22] respectively, members of the baseline lineage were still detected, however, alongside new lineages.

In some instances, it appears the variation in the population is seeded from another population. For example, as shown for CV-B3 and E7 (Figs. 2 and 5), it appears that in both instances, strains from Asia were imported into Nigeria and subsequently detected in the faeces of children diagnosed with AFP. These suspected 
NGR LG2012 IJR 24 E7

NGR LG2012 IJR 29 E7

NGR LG2012 IJR 25 E7

97 NGR LG2012 IJR 27 E7

NGR LG2012 IJR 26 E7

NGR LG2012 IJR 28 E7

NGR LG2012 IJR 30 E7

— NGR LG2012 IJR 11 E7

— E 7|KM303089|NIG 201009 E7 LAG IJR AUG 2010

—E 7|KM303087|NIG 201006 E7 LAG IYNPJ AUG 2010

E 7|KM303082|NIG 201001 E7 LAG UNLG JULY 2010

63 E 7|KM264408|NIG IB 201007

— E 7|KM303083|NIG 201002 E7 LAG MKK JULY 2010

$100 \checkmark$ C5 E7 AFP NGR 2014

53

C8 E7 AFP NGR 2014

. NGR LG2013 IJR P5 E7

99 NGR LG2013 IJR P7 E7

57 NGR LG2013 IJR P9 E7

KY433619.1 E7 13704 AFP NIGER 2013

60 KY433618.1 E7 13680 AFP NIGER 2013

91 KY433706.1 E7 13555 AFP NIGER 2013

E 7|JN255656.1| CAF-OUP-06-156 2006

92 E 7|JN255650.1| CAF-BAN-06-122 2006

99 E 7|JN255653.1| CAF-NMA-06-018 2006

E 7|JN255655.1| CAF-OUH-01-053a 2001

100 E 7JJN255652.1| CAF-BKO-01-006 2001

E 7JJN255654.1| CAF-OUA-01-046 2001

- E 7|JN255651.1| CAF-BAN-98-022 1998

\begin{tabular}{|c|c|c|c|c|c|c|c|}
$57-$ KY433616.1 E7 13676 AFP SENEGAL 2013 & D \\
\hline KY433617.1 E7 13 677 AFP SENEGAL 2013
\end{tabular}

KY433620.1 E7 13 704 AFP NIGER 2014

$62 \quad$ KY433621.1 E7 14427 AFP MAURITANIA 2014 E

99- KY433622.1 E7 14641 AFP MAURITANIA 2014

24 BANGLADESH AND CHINA 2007-2010

E 7|JX513460.1|A171D INDIA 2008

GEORGIA, INDIA \&: CHINA, 2002 2010

EUROPE, ASIA \& AUSTTRALLIA

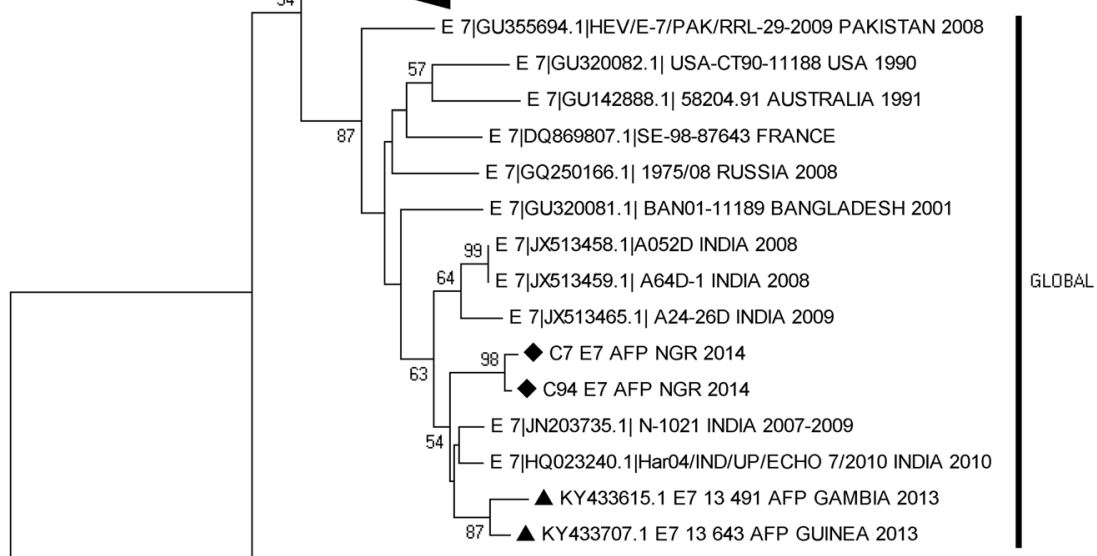

E $7 \mid A Y 302559$ STRAIN WALLACE

- NGR LG2012 12 E33

Fig. 5 (See legend on next page.) 
(See figure on previous page.)

Fig. 5 Phylogram of genetic relationship between VP1 nucleotide sequences of E7 isolates. The phylogenetic tree is based on an alignment of the partial VP1 sequences. The newly sequenced strains are indicated with black diamond while other strains from west-Africa within the Global cluster are indicated with black triangle. The GenBank accession numbers and strain of the isolates are indicated in the tree. Bootstrap values are indicated if $>50 \%$

importations corroborate what is known about poliovirus global circulation $[8,29]$. What is not clear is why, as suggested in the regional confinement hypothesis [30], most non-polio enterovirus lineages detected in sub-Saharan Africa are yet to be detected and described in other world regions. Also interesting is the observation that enterovirus lineages found in Nigeria are also present and circulating in Niger (Figs. 2, 4 \& 5). Considering the apparent porosity of borders in the Chad basin, this is not surprising. It is however crucial to note that the lineages circulating in Nigeria and Niger appear to be different from those recently shown [31] to be circulating in Gambia, Guinea and Senegal (Figs. 3, 4 \& 5). Hence, though all the mentioned countries are in subSaharan Africa and more interestingly, West-Africa, it appears there might also be a level of sub-regional restriction of circulation. Characterization of more NPEV isolates will definitely show whether the observed is a true biological phenomenon or relic of paucity of data.

In this study, three (one $5^{\prime}$-UTR and two VP1) different enterovirus detection assays were used. The $5^{\prime}$-UTR screen is used to screen isolates in a bid to determine if they are enteroviruses. This is done because the 5'untranslated region (UTR) contains the internal ribosome entry site (IRES). On entry into the cytoplasm of any susceptible cell, to initiate translation of the single open reading frame (ORF) in the enterovirus genome, the ribosome must be assembled on the IRES in the $5^{\prime}$ UTR. The consequent conservation of this enterovirus genomic region is capitalized upon for enterovirus detection using the $5^{\prime}$-UTR assay. However, because of the conserved nature of the $5^{\prime}$-UTR its sequences do not provide the resolution needed to determine enterovirus type. On the other hand, the VP1 gene encodes one of the virus structural proteins. Studies [1-6] have established a correlation between the nucleotide or amino acid sequence of VP1 and enterovirus types determined using neutralization assays. The fact that there are over two hundred and fifty (250) enterovirus types (www.picornaviridae.com) gives a good impression of how variable the VP1 structural protein is. Hence, irrespective of how degenerate the primer combinations used, it might not be surprising if any one Panenterovirus RT-PCR screen fails to amplify the VP1 gene of any enterovirus of interest. Against this backdrop, enterovirus identification assays (including the poliovirus identification algorithm in use by the Global Polio
Eradication Initiative [GPEI]) are built around first detecting the conserved enterovirus genomic region $\left(5^{\prime}\right.$ UTR) and subsequently subjecting isolates detected by this screen to VP1 assays [4-6].

Overall, six (6/96) of the isolates analyzed were positive for the $5^{\prime}$-UTR screen but negative for the VP1 screen. These isolates might therefore be enteroviruses that have VP1 primer binding sites that are too divergent to be bound by the primers used in this study. Another seven (7/96) isolates on the other hand were negative for the $5^{\prime}$-UTR screen but positive for the VP1 assays. Should we have used the $5^{\prime}$-UTR result as the basis for selecting isolates subjected to the VP1 screens, all these isolates would have been missed. It is currently not clear why these isolates were negative for the $5^{\prime}$-UTR screen considering they replicated in culture and are consequently viable strains. However, it is important to mention that recently, a new enterovirus $5^{\prime}$-UTR region was described that has a large deletion overlapping the region amplified in this assay [32-35]. Though described in EV-C isolates, it is not clear whether such constructs are present in members of other enterovirus species and whether such constructs would be functional when transferred to other species through recombination in the $5^{\prime}$-UTR region. Whatever be the case, the $5^{\prime}$-UTR primers used in this study could not detect these isolates. Consequently, the results of this study suggest that coupling the 5 '-UTR and VP1 assays in a way that ensures both are independent and equally important for enterovirus identification might provide the added sensitivity required to find some divergent types. Particularly, it suggests that being positive for the $5^{\prime}$-UTR assay should not be the basis for subjecting isolates to the VP1 assays.

In this study, there were different groups of 'untypable' isolates (Table 1). The first group were those positive for the $5^{\prime}$-UTR screen but negative for the VP1 screen and have been addressed above. The second group were those positive for the VP1 assay but for which the eletropherogram could not be exploited due to multiple peaks. These are likely to come from cases where the children in question were co-infected with more than one type of enterovirus. This is not unusual and, as previously mentioned, we have more recently observed (unpublished) that in about $50 \%$ of cases, stool samples from children with AFP in Nigeria contain more than one enterovirus type and/or species. 
The third group were those negative for both the $5^{\prime}$ UTR and the VP1 assays. Considering we detected isolates that were positive for the $5^{\prime}$-UTR screen but negative for the VP1 screen and vice-versa, it is not difficult to conceptualize the possibility that enterovirus isolates might exist that are negative for both assays. However, currently, this is only a conjecture. Since RD cell line can also support replication of other enteric viruses like the adenoviruses [36], these third group of 'untypables' might not necessarily be enteroviruses but could be other viruses for which the RD cell line is both susceptible and permissive.

\section{Conclusions}

We identified 27 different enterovirus types present in Nigerian children with AFP in 2014 and document the first molecular detection/identification 9 Nigerian enterovirus strains, thereby expanding the catalogue of enterovirus types circulating and probably contributing to AFP in the region. There might also be importation into Nigeria of enterovirus clades from other world regions, and especially Asia. The study also shows that coupling the $5^{\prime}$-UTR and VP1 assays in a way that ensures both are independent and equally important for enterovirus identification might provide some of the added sensitivity required to detect more divergent enterovirus types. We therefore suggest revision of the current identification algorithm for enteroviruses and also surveillance of the environment, AFP and healthy children, as these have provided new information and may contribute significantly towards obtaining a complete picture of the diversity of enterovirus types present and circulating in any population.

\section{Abbreviations \\ AFP: Acute flaccid paralysis; cDNA: complementary Deoxyribonucleic Acid; CPE: Cytopathic effect; CV: Coxsackievirus; E: Echovirus; ES: Environmental surveillance; EV: Enterovirus; F: Forward; GPEl: Global Polio Eradication Initiative; GPLN: Global Polio Laboratory Network; IPV: Inactivated Polio Vaccine; L20B: Genetically engineered mouse cell line expressing the human cell surface receptor for poliovirus; MCF-7: Breast Cancer cell line; NPEV: Non- polio enterovirus; OPV: Oral Polio Vaccine; PCR: Polymerase chain reaction; PE: PanEnterovirus; R: Reverse; RD: Human rhabdomyosarcoma; RNA: Ribonucleic acid; UTR: Untranslated region; VP1: Virus Protein 1; WHA: World Health Assembly; WHO: World Health Organization}

\section{Acknowledgements}

We thank the WHO National Polio Laboratory in Ibadan, Nigeria for providing the anonymous isolates analyzed in this study.

\section{Funding}

This study was funded by a Tertiary Education Trust Fund, Research Project Intervention, Abuja, Nigeria to FO. The funding body had no role in study design; in the collection, analysis and interpretation of data; in the writing of the report; and in the decision to submit the article for publication. Hence, the opinions expressed in this article are solely those of the authors.

\section{Availability of data and materials}

The data presented in this manuscript are therein. Those not presented have been submitted to and can be recovered from GenBank.

\section{Authors' contributions}

FTOC, AMO, AJA: sample collection and laboratory analysis. FTOC: wrote the first draft of the manuscript. AJA and FO both supervised this study. All authors: study design, data analysis, revised the manuscript, read and approved the final draft.

\section{Ethics approval and consent to participate}

Enterovirus isolates were analysed in this study. The stool samples from which these isolates were recovered were collected in accordance with the national ethical guidelines as part of the National AFP surveillance programme in Nigeria and sent to the WHO National Polio Laboratory in Ibadan, Nigeria to ascertain whether poliovirus is the etiologic agent of the diagnosed AFP using the WHO algorithm. The isolates analyzed in this study were subsequently anonymized for further studies before use in this study. Thus, this article does not contain any studies with human participants performed by any of the authors. In addition, no information that can be used to associate the isolates analyzed in this study to any individual is included in this manuscript.

\section{Consent for publication}

Not applicable.

\section{Competing interests}

The authors declare that no conflict of interests exist.

\section{Publisher's Note}

Springer Nature remains neutral with regard to jurisdictional claims in published maps and institutional affiliations.

\section{Author details}

'Department of Microbiology, Faculty of Science, Ekiti State University, Ado-Ekiti, Ekiti State, Nigeria. '2Department of Virology, College of Medicine, University of Ibadan, Ibadan, Oyo State, Nigeria. ${ }^{3}$ Department of Microbiology, Faculty of Science, Obafemi Awolowo University, Ile-lfe, Osun State, Nigeria. ${ }^{4}$ WHO, National Polio Laboratory, Department of Virology, College of Medicine, University of Ibadan, Ibadan, Oyo State, Nigeria. ${ }^{5}$ Microbiology Unit, Department of Biological Sciences, Kings University, P.M.B. 555, Odeomu, Osogbo, Osun State, Nigeria.

Received: 26 March 2017 Accepted: 5 September 2017

Published online: 12 September 2017

\section{References}

1. Oberste MS, Maher K, Kilpatrick DR, Pallansch MA. Molecular evolution of the human enteroviruses: correlation of serotype with VP1 sequence and application to picornavirus classification. J Virol. 1999;73(3):1941-8,

2. Oberste MS, Nix WA, Maher K, Pallansch MA. Improved molecular identification of enteroviruses by RT-PCR and amplicon sequencing. J Clin Virol. 2003;26(3):375-7.

3. Oberste MS, Maher K, Williams AJ, Dybdahl-Sissoko N, Brown BA, Gookin MS, Penaranda S, Mishrik N, Uddin M, Pallansch MA. Species-specific RT-PCR amplification of human enteroviruses: a tool for rapid species identification of uncharacterized enteroviruses. J Gen Virol. 2006;87:119-28.

4. Oberste MS, Pallansch MA. Enteroviruses molecular detection and typing. Reviews in Medical Microbiology. 2005;16:163-71.

5. Nix WA, Oberste MS, Pallansch MA. Sensitive, Seminested PCR amplification of VP1 sequences for direct identification of all Enterovirus serotypes from original clinical specimens. J Clin Microbiol. 2006;44(8):2698-704.

6. World Health Organisation. Enterovirus surveillance guidelines: Guidelines for enterovirus surveillance in support of the Polio Eradication Initiative. Geneva: WHO; 2015

7. WHO 1988. Global eradication of poliomyelitis by the year 2000 (World Health Assembly resolutionWHA41-28) http://polioeradication.org/wpcontent/uploads/2016/07/19880513_resolution-2.pdf.

8. Nathanson N, Kew OM. From emergence to eradication: the epidemiology of poliomyelitis deconstructed. Am J Epidemiol. 2010;172(11):1213-29.

9. Minor PD. The polio-eradication programme and issues of the end game. J Gen Virol. 2012;93:457-74.

10. Ranta J, Hovi T, Arjas E. Poliovirus surveillance by examining sewage water specimens: studies on detection probability using simulation models. Risk Anal. 2001;21(6):1087-96. 
11. World Health Organisation. Guidelines for environmental surveillance of poliovirus circulation. Geneva: WHO; 2003.

12. World Health Organisation. Polio laboratory Manual. 4th Edition, Geneva: WHO; 2004.

13. McAllister RM, Melnyk J, Finklestein JZ, Adams EC, Gardner MB. Cultivation in vitro of cells derived from a human rhabdomyosarcoma. Cancer. 1969;24:520-6.

14. Pipkin PA, Wood DJ, Racaniello VR, Minor PD. Characterization of $L$ cells expressing the human poliovirus receptor for the specific detection of polioviruses in vitro. J Virol Methods. 1993;41:333-40.

15. Yoshii K, Yoneyama T, Shimizu H, Yoshida H, Hagiwara A. Sensitivity of cells to poliovirus. Japanese Journal of Infectious Disease. 1999;52(4):169.

16. Oyero OG, Adu FD. Non-polio enteroviruses serotypes circulating in Nigeria. Afr J Med Med Sci. 2010;39(Supple):201-8.

17. Oyero OG, Adu FD, Ayukekbong JA. Molecular characterization of diverse species enterovirus-B types from children with acute flaccid paralysis and asymptomatic children in Nigeria. Virus Res. 2014;189:189-93.

18. Adeniji JA, Faleye TOC. Isolation and identification of enteroviruses from sewage and sewage contaminated water in Lagos, Nigeria. Food Environ Virol. 2014a;6(2):75-86.

19. Adeniji JA, Faleye TOC. Impact of cell lines included in Enterovirus isolation protocol on perception of nonpolio Enterovirus species C diversity. J Virol Methods. 2014b;207:238-47.

20. Adeniji JA, Faleye TOC. Enterovirus C strains circulating in Nigeria and their contribution to the emergence of recombinant circulating vaccine-derived polioviruses. Arch Virol. 2015;160(3):675-83.

21. Faleye TOC, Adeniji JA. Nonpolio Enterovirus-C (NPEV-C) strains circulating in south-western Nigeria and their contribution to the emergence of recombinant cVDPV2 lineages. British Journal of Virology. 2015a;2(5):68-73.

22. Faleye TOC, Adeniji JA. Enterovirus species B bias of RD cell line and its influence on Enterovirus diversity landscape. Food Environ Virol. 2015b;7(4):390-402

23. Faleye TOC, Adewumi MO, Coker BA, Nudamajo FY, Adeniji JA. Direct detection and identification of enteroviruses from faeces of healthy Nigerian children using a cell-culture independent RT- semi-nested PCR assay. Advances in Virology. 2016;2016:1412838. doi:10.1155/2016/1412838.

24. Kroneman A, Vennema H, Deforche K, v d Avoort H, Penarandac S, Oberste MS, Vinjéc J, Koopmans M. An automated genotyping tool for enteroviruses and noroviruses. J Clin Virol. 2011;51:121-125.

25. Tamura K, Peterson D, Peterson N, Stecher G, Nei M, Kumar S. MEGA5: Molecular evolutionary genetics analysis using maximum likelihood, evolutionary distance, and maximum parsimony methods. Mol Biol Evol. 2011;28(10):2731-9.

26. Kimura M. A simple method for estimating evolutionary rate of base substitutions through comparative studies of nucleotide sequences. J Mol Evol. 1980;16(2):111-20.

27. Baba MM, Oderinde BS, Patrick PZ, Jarmai MM. Sabin and wild polioviruses from apparently healthy primary school children in northeastern Nigeria. J Med Virol. 2012;84(2):358-64.

28. Adeniji JA, Oragwa AO, George UE, Ibok UI, Faleye TOC, Adewumi MO. Preponderance of Enterovirus species C in RD-L20B cell culture negative stool samples from children diagnosed with acute flaccid paralysis in Nigeria. Arch Virol. 2017; doi:10.1007/s00705-017-3466-2.

29. Burns CC, Diop OM, Sutter RW, Kew OM. Vaccine derived polioviruses. Journal of Infectious Disease. 2014;210(Suppl 1):S283-93.

30. Sadeuh-Mba SA, Bessaud M, Massenet D, Joffret ML, Endegue MC, Njouom R, Reynes JM, Rousset D, Delpeyroux F. High frequency and diversity of species $C$ enteroviruses in Cameroon and neighboring countries. J Clin Microbiol. 2013:51(3):759-70.

31. Fernandez-Garcia MD, Kebe O, Fall AD, Ndiaye K. Identification and molecular characterization of non-polio enteroviruses from children with acute flaccid paralysis in West-Africa, 2013-2014. Sci Rep. 2017;7:3808. doi:10.1038/s41598-017-03835-1.

32. Tapparel C, Junier T, Gerlach D, Van-Belle S, Turin L, Cordey S, et al. New respiratory enterovirus and recombinant rhinoviruses among circulating picornaviruses. Emerg Infect Dis. 2009;15:719-26.

33. Yozwiak NL, Skewes-Cox P, Gordon A, Saborio S, Kuan G, Balmaseda A, et al. Human enterovirus 109: a novel interspecies recombinant enterovirus isolated from a case of acute pediatric respiratory illness in Nicaragua. J Virol. 2010;84:9047-58.
34. Lukashev AN, Drexler JF, Kotova VO, Amjaga EN, Reznik VI, Gmyl AP, Grard G, Taty T, Trotsenko OE, Leroy EM, Drosten C. Novel serotypes 105 and 116 are members of distinct subgroups of human enterovirus $C$. J Gen Virol. 2012:93:2357-62.

35. Richter J, Tryfonos C, Panagiotou C, Nikolaou E, Maria Koliou M, Christodoulou C. Newly emerging C group enteroviruses may elude diagnosis due to a divergent 5'-UTR. Int J Infect Dis. 2013;17(12):e1245-8.

36. Enomoto M, Fujimoto T, Konagaya M, Hanaoka N, Chikahira M, Taniguchi K, Okabe N. Cultivation for 21 days should be considered to isolate respiratory adenoviruses from samples containing small numbers of adenoviral genomes. Jpn J Infect Dis. 2010;63(5):338-41.

\section{Submit your next manuscript to BioMed Central and we will help you at every step:}

- We accept pre-submission inquiries

- Our selector tool helps you to find the most relevant journal

- We provide round the clock customer support

- Convenient online submission

- Thorough peer review

- Inclusion in PubMed and all major indexing services

- Maximum visibility for your research

Submit your manuscript at www.biomedcentral.com/submit
Biomed Central 